\title{
Biochemical and photochemical analyses of retinal proteins in chicken cone cells
}

\author{
Tôru Yoshizawa, Yoshinori Shichida and Yoshitaka Fukada \\ Department of Biophysics, Faculty of Science, Kyoto University, \\ Kyoto 606, Japan
}

\begin{abstract}
Absorption of light by several types of cone cell with different spectral sensitivities initiates in vertebrates photopic vision which includes color discrimination. In order to understand the conversion mechanism of a photon signal in the cones, photochemical reactions of lodopsin, the red-sensitive cone visual pigment of chicken, and its interaction with a G-protein were investigated and compared with those of rhodopsin, the visual pigment in rod cells that are responsible for scotopic vision. The results obtained indicate that the photobleaching processes of both pigments and the signal transduction processes in the two types of the cell are similar to, but somewhat different from each other. This could partly explain the differences between the physiological responses of the different cells.
\end{abstract}

\section{INTRODUCTION}

The retina of vertebrates contains two kinds of photoreceptor cells, rods and cones, which were first distinguished from each other on the basis of the morphological shape of their outer segments. Afterwards, it was confirmed that the rod and cone are responsible for scotopic and photopic vision, respectively. In the human retina, there are at least three types of cone, each of which has a visual pigment with a distinctive absorption maximum. The integration of the photoresponses from the cones finally generates the sensation of color, in the visual cortex.

Capture of a photon by a visual pigment molecule in the outer segment can initiate an enzymic cascade reaction in the photoreceptor cell. Vertebrate visual pigments contain an 11-cis retinylidene chromophore bound via a protonated Schiff base linkage to a specific lysine residue of the apoprotein, opsin. The primary event in the functioning of rhodopsin, the rod visual pigment, has been extensively studied and shown to be an isomerization around the $C(11)=C(12)$ double bond of the chromophore to form a twisted all-trans photoproduct, photorhodopsin (ref. 1). The subsequent thermal reactions proceed through several intermediates to form an active intermediate, metarhodopsin 11 . This intermediate can bind to transducin, a guanine nucleotide-binding protein, to convert it into an active form (GTP-bound form) by which cGMP-phosphodiesterase can be activated. The hydrolysis of cGMP in the outer segment changes the cation channel in the plasma membrane of the outer segment from an open to a closed state. This generates the receptor potential. These investigations have been conducted excusively in rod cells.

The rod is a photoreceptor cell which, during evolution, has acquired an extremely high photosensitivity, so that it can act under twilight conditions. The cone, however, acts under daylight conditions; its photoresponse is wider in dynamic range and faster in generation than that of the rod. Little is known about the molecular mechanism of the photo-transduction process in the cone. One of the reasons for this is the difficulty of purifying cone visual pigments. The only cone pigment which has been extracted and investigated biochemically is iodopsin, a red-sensitive cone pigment from chicken retina.

The chemical nature of lodopsin is compared with that of rhodopsin in Table 1. Like rhodopsin, iodopsin has (11-cis)-retinal as its prosthetic group (ref. 2). The absorption maximum of iodopsin extracted with digitonin is at $562 \mathrm{~nm}$ or $512 \mathrm{~nm}$ in the presence or absence of chloride ion, respectively (ref. 3,4$)$. The rate of regeneration of iodopsin from (11-cis)retinal and R-photopsin (the protein moiety of iodopsin) is much faster than that of rhodopsin from (11-cis)-retinal and scotopsin (the protein moiety of rhodopsin) (ref. 2). Unlike rhodopsin, iodopsin is bleached in the presence of simple chemical reagents such as as $\mathrm{NH}_{2} \mathrm{OH}_{\text {, }}$ alum (ref. 2) or $\mathrm{NaBH}_{4}$ (ref. 5). Interestingly, the addition of (9-cis)-retinal to an lodopsin extract causes a chromophore exchange reaction, which results in formation of (9-cis)-iodopsin (ref. 5). Furthermore, when iodopsin coexists with scotopsin in the extract, the (11-cis) chromophore of iodopsin transfers to the scotopsin, resulting in the formation of rhodopsin (ref. 5). 
TABLE 1. Comparison between Properties of Chicken lodopsin and Rhodopsin.

\begin{tabular}{|c|c|c|c|}
\hline & Rhodopsin & lodopsin & \\
\hline Chromophore & (11-cis)-Retinal & (11-cis)-Retinal & [ref. 2] \\
\hline Protein moiety & Scotopsin & R-Photopsin & [ref. 2] \\
\hline $\begin{array}{c}\text { Absorption maximum } \\
\left(\lambda_{\max }\right)\end{array}$ & $501 \mathrm{~nm}$ & (in the presence of $\mathrm{Cl}^{-}$) & [ref. 2] \\
\hline $\begin{array}{c}\text { Chloride effect } \\
\text { on } \lambda_{\max }\end{array}$ & No effect & $\begin{array}{c}512 \mathrm{~nm} \\
\text { (in the absence of } \mathrm{Cl}^{-} \text {) }\end{array}$ & [ref. 3, 4] \\
\hline $\begin{array}{l}\text { Relative regeneration } \\
\text { rate at } 10^{\circ} \mathrm{C}\end{array}$ & 1 & 527 & [ref. 2] \\
\hline Stable in $\mathrm{pH}$ region of & $4-9$ & $5-7$ & [ref. 2] \\
\hline In $\mathrm{NH}_{2} \mathrm{OH}$ & Stable & Bleached & [ref. 2] \\
\hline In Alum & Stable & Bleached & [ref. 2] \\
\hline In $\mathrm{NaBH}_{4}$ & Stable & Bleached & [ref. 5] \\
\hline $\begin{array}{l}\text { Replacement of } \\
\text { chromophore }\end{array}$ & Not replaced & $\begin{array}{r}\text { Replaced by } \\
(9-\text { cis)-retinal }\end{array}$ & [ref. 5] \\
\hline $\begin{array}{l}\text { Transfer of } \\
\text { chromophore }\end{array}$ & Not transferred & $\begin{array}{l}\text { Transferred to } \\
\text { scotopsin }\end{array}$ & [ref. 5] \\
\hline
\end{tabular}

Besides these differences between rhodopsin and iodopsin, it is worthwhile noting the differences in photoresponse between rod and cone (ref. 6). The dynamic range of photoresponse in the cone is about $10^{3}$ times wider than that in the rod and the rate of generation of photoresponse in the cone is about 4 times faster than that in the rod. Furthermore, the photosensitivity of the rod is about 100 times greater than that of the cone. In order to try to elucidate these differences of the molecular level, we have started to investigate the biochemical and photochemical properties of lodopsin, and compared them with those of bovine rhodopsin.

\section{PURIFICATION OF CHICKEN CONE PIGMENTS}

Although cone visual pigments are much lower in stability than rhodopsin, we have succeeded in extracting them from chicken photoreceptor membranes by using $0.75 \% \mathrm{CHAPS}$ as an extraction buffer. We found that the addition of $1.0 \mathrm{mg} / \mathrm{ml}$ egg yolk phosphatidyl-choline (PC) to the extraction buffer markedly stabilized the cone pigments. A visual pigment preparation extracted from the chicken photoreceptor membranes by the CHAPS-PC buffer was subjected to successive column chromatography on Con A- and DEAE-Sepharose to purify iodopsin and rhodopsin (Fig. 1) (ref. 7). The molecular masses of iodopsin and rhodopsin were estimated to be $36 \mathrm{kDa}$ and $37 \mathrm{kDa}$, respectively, by SDS-polyacrylamide gel electrophoresis. Besides these two pigments, three other kinds of cone pigment, green-, blue-, and violet-sensitive pigments, were identified in the extract by spectrophotometric measurements. The absorption maxima of the pigments were determined as follows: rhodopsin, $503 \mathrm{~nm}$; iodopsin, $571 \mathrm{~nm}$; green; $508 \mathrm{~nm}$; blue, $455 \mathrm{~nm}$; violet, $415 \mathrm{~nm}$ (Fig. 2). These values were in good agreement with those determined microspectrophotometrically rather than those reported in digitonin, suggesting that the conformations of pigments in the CHAPS-PC system may be closer than those in digitonin to the native ones.

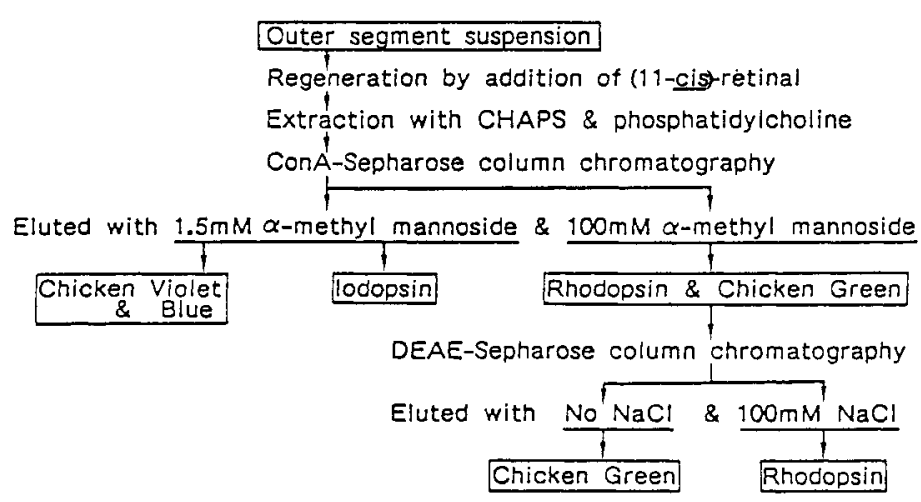

Fig. 1. Procedures for isolating rod and cone visual pigments from chicken outer segment membranes. After regeneration of all the pigments by the addition of (11cis)-retinal, all the procedures were carried out in complete darkness or under dim red light. 


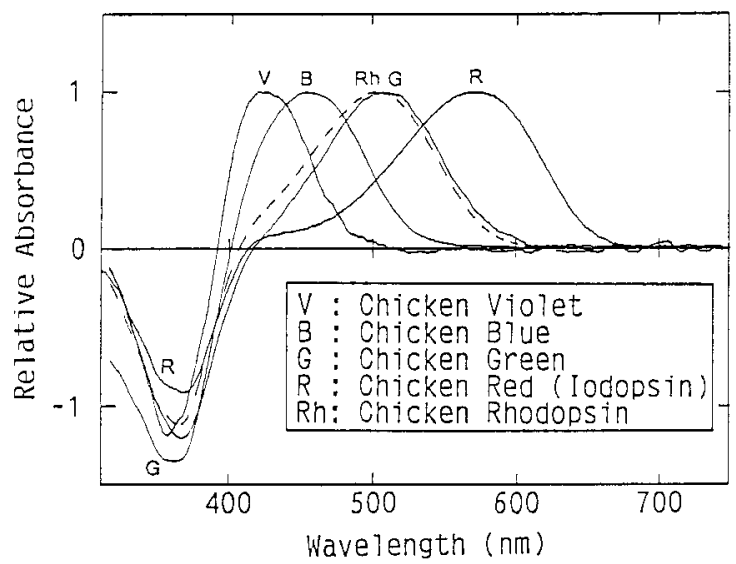

Fig. 2. Difference spectra of five kinds of chicken visual pigments relative to their retinal-oxime plus opsins. Except for chicken green, the spectra are the difference before and after complete photobleaching in the presence of $10 \mathrm{mM} \mathrm{NH} \mathrm{NH}_{2} \mathrm{OH}$. The spectrum of chicken green is the difference before and after thermal-bleaching in the presence of $10 \mathrm{mM} \mathrm{NH}_{2} \mathrm{OH}$. All the spectra were measured at $4^{\circ} \mathrm{C}$.

\section{RETINAL-ABINDING SITE OF IODOPSIN}

The purified iodopsin was completely bleached in the presence of $10 \mathrm{mM} \mathrm{NH}_{2} \mathrm{OH}$, from which R-photopsin free from retinal-oxime was isolated by Con A-Sepharose column chromatography. Scotopsin was similarly prepared from purified bovine rhodopsin. In the presence of chloride ion, the retinal-binding sites of both the opsins were examined by the use of geometric isomers of retinal (13-cis, 11-cis, 9-cis, 7-cis and all-trans retinals) and retinal analogs including fluorinated $(14-\mathrm{F}, 12-\mathrm{F}, 10-\mathrm{F}$, and $8-\mathrm{F})$ and methylated (12-Me) (11-cis)-retinals (ref, 8). The stereoselectivity of R-photopsin for the retinal isomers and analogs was almost identical to that of scotopsin, indicating that the shape of the retinal-binding site of both opsins is somewhat similar. The rates of pigment formation from R-photopsin were considerably higher than those from scotopsin. In addition, all the lodopsin isomers and analogues were more susceptible to $\mathrm{NH}_{2} \mathrm{OH}$ than were the rhodopsin ones. From these observations, the retinal-binding site of iodopsin is inferred to be located near the protein surface as we had suggested previously (ref. 5). On the basis of the opsin shift of fluorinated analogs (Table 2), a polar group in the retinal-binding site of iodopsin, as in rhodopsin, would be located near the hydrogen atom at the $\mathrm{C}-10$ position of the retinylidene chromophore. The large difference between the absorption maxima of lodopsin and rhodopsin was significantly reduced in the 9-cis and 7-cis pigments. On the assumption that the retinylidene chromophore is anchored rigidly at the $\alpha$-carbon of the lysine residue and loosely at the cyclohexenyl ring (ref. 9), the 9-cis and 7-cis isomers would have the Schiff-base nitrogen at a position altered from that of the 11-cis pigment. Thus, the remarkable red-shift in the absorption spectrum of lodopsin compared with that of rhodopsin could be attributed to a difference between the two pigments in the location of a negative charge directly hydrogen-bonded to the Schiff-base nitrogen. Our simplest model is that iodopsin would have a Schiff-base weakly hydrogen-bonded to the counter-ion and a second point charge at a position similar to that in rhodopsin (Fig. 3).

Table 2. Absorption Maxima of Pigments and Their Differences in Wavenumber.

\begin{tabular}{|c|c|c|c|c|c|c|}
\hline \multirow{2}{*}{$\begin{array}{l}\text { Retinal } \\
\text { isomer } \\
\text { and } \\
\text { analog }\end{array}$} & \multirow{2}{*}{$\begin{array}{c}\text { lodopsin } \\
\text { CHAPS-PCa } \\
(\mathrm{nm})\end{array}$} & \multirow{2}{*}{$\begin{array}{c}\text { Rhodopsin } \\
\text { CHAPS-PCa } \\
(\mathrm{nm})\end{array}$} & \multirow{2}{*}{$\begin{array}{l}\frac{\mathrm{PSB}^{\mathrm{b}}}{\mathrm{MeOH}} \\
(\mathrm{nm})\end{array}$} & \multicolumn{3}{|c|}{ Difference in wavenumber } \\
\hline & & & & $\begin{array}{l}\text { PSB-lodC } \\
\left(\mathrm{cm}^{-1}\right)\end{array}$ & $\begin{array}{l}\text { PSB-Rhd } \\
\left(\mathrm{cm}^{-1}\right)\end{array}$ & $\begin{array}{r}\text { Rh-lode } \\
\left(\mathrm{cm}^{-1}\right)\end{array}$ \\
\hline 11 -cis & 571 & 500 & 442 & 5111 & 2624 & 2487 \\
\hline 9 -cis & 534 & 484 & 433 & 4368 & 2434 & 1935 \\
\hline 7 -cis & 463 & 451 & 432 & 1550 & 975 & 574 \\
\hline $14-\bar{F}$ & 607 & 527 & 455 & 5504 & 3003 & 2501 \\
\hline $12-F$ & 586 & 507 & 446 & 5357 & 2698 & 2659 \\
\hline $10-\mathrm{F}$ & 574 & 499 & 428 & 5943 & 3324 & 2619 \\
\hline 8-F & 514 & 463 & 410 & 4935 & 2792 & 2143 \\
\hline $12-\mathrm{Me}$ & 534 & 507 & 440 & 4001 & 3003 & 997 \\
\hline
\end{tabular}

aDetermined in $0.6 \%$ CHAPS containing $0.8 \mathrm{mg} / \mathrm{ml}$ egg yolk phosphatidylcholine (PC). bAbsorption maximum of a protonated retinylidene Schiff-base (PSB) in methanol. CFor each isomer or analog of retinal, the difference in absorption maximum between the PSB and the pigment with R-photopsin was calculated on a wavenumber scale. $\underline{d} A s \underline{c}$, but the difference between the PSB and the pigment with scotopsin. $\frac{e}{s} \bar{s}$, but the difference between the pigments with scotopsin and with R-photopsin. 


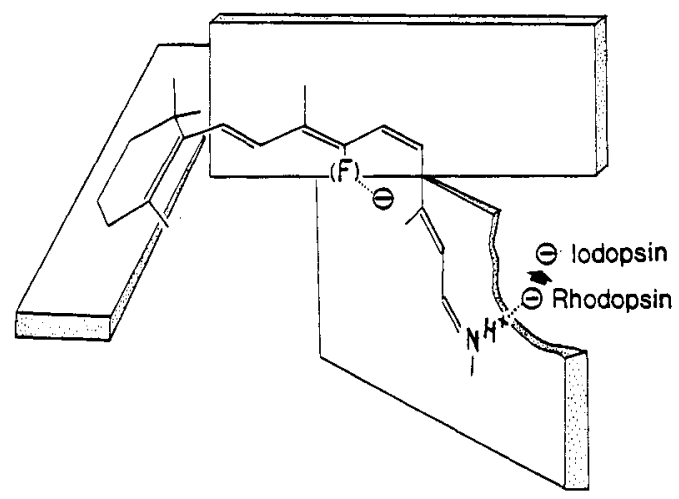

Fig. 3. A model of the chromophore in iodopsin. A negative counter-ion to the protonated Schiff-base nitrogen in iodopsin is placed slightly further away than that in rhodopsin. A second negative charge in iodopsin is placed at a position similar to that in rhodopsin near the $\mathrm{C}-13$ position so as to interact with the fluorine atom of 10-F-retinal.

\section{EFFECT OF CHLORIDE ION ON THE ABSORPTION MAXIMUM OF IODOPSIN}

As already shown in Table 1 , one of the most prominent chemical differences between iodopsin and rhodopsin is that the absorption spectrum of iodopsin is affected by chloride ion. Our recent experiments on the chloride-dependent spectral shift of iodopsin revealed that iodopsin has a single chloride-binding site in its protein moiety with a dissociation constant of $0.8 \mathrm{mM}$ chloride (ref. 10). The binding site accepts other monovalent anions, e.g. $\mathrm{NO}_{3}^{-}$, but not divalent anions such as $\mathrm{SO}_{4}{ }^{2-}$. It seems probable that there may exist an equilibrium between chloride-bound form of iodopsin and its depleted form. Interestingly, we observed that the chromophore of chloride-depleted iodopsin was easily transferred to scotopsin in the extract, resulting in the formation of rhodopsin. However, the chromohore transfer reaction was inhibited by the binding of chloride in iodopsin (ref. 10). Since the (11-cis)-retinylidene chromophore of rhodopsin is embedded in the center of the $\alpha$-helix region of rhodopsin, it is unlikely that (11-cis)-retinal was captured by scotopsin through direct contact with iodopsin. Therefore, the results indicate that the chloride-depleted lodopsin is partly dissociated into (11-cis)-retinal and R-photopsin as shown in Fig. 4. This reaction scheme can also explain the accessibility of iodopsin to various chemicals including $\mathrm{NH}_{2} \mathrm{OH}$. Besides regulating the absorption spectrum of lodopsin, the chloride stabilizes the binding of the chromophore in the protein.

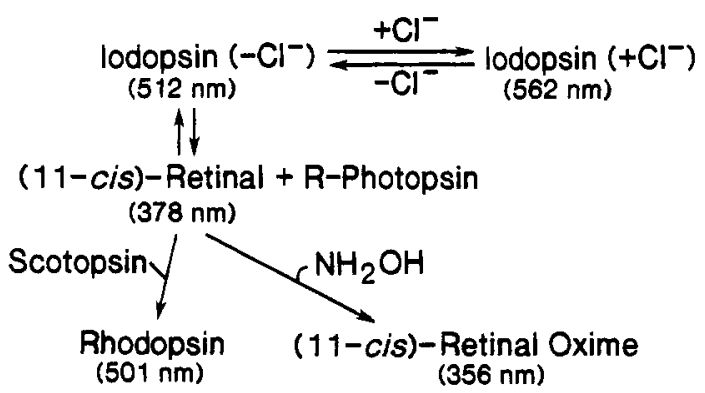

Fig. 4. A scheme for the reactions of lodopsin in the presence of scotopsin or $\mathrm{NH}_{2} \mathrm{OH}$.

\section{BATHOIDOPSIN AND CHLORIDE ION}

Since the binding of chloride stabilizes the chromophore-protein interactions in iodopsin, it would affect the photobleaching process of iodopsin. The photobleaching process of iodopsin has so far been investigated only by low temperature spectrophotometry. A few intermediates were found in the bleaching process. Yoshizawa and Wald (ref. 11) observed formation of batholodopsin by irradiation of iodopsin at liquid nitrogen temperature. They described a peculiar phenomenon, that bathoiodopsin did not convert thermally to the next intermediate, lumiiodopsin, but was reverted to the original iodopsin on warming above $-180^{\circ} \mathrm{C}$, though bathorhodopsin was thermally converted to the next intermediate, lumirhodopsin. In order to check whether or not the thermal back reaction of bathoiodopsin to iodopsin is affected by the binding of chloride to the protein moiety of iodopsin, we have investigated the photobleaching process of iodopsin where the chloride-binding site was occupied by nitrate (denoted $\mathrm{N}$-iodopsin).

Irradiation of $\mathrm{N}$-iodopsin at liquid nitrogen temperature led to formation of the bathointermediate of $\mathrm{N}$-iodopsin. On warming above $-160^{\circ} \mathrm{C}$, this was converted into the next intermediate, lumi-intermediate, before bleaching occurred to (all-trans)-retinal and Rphotopsin (Fig. 5). Thus the binding of chloride to the protein moiety of iodopsin changes the photobleaching process of iodopsin (ref. 12). 


\begin{tabular}{|c|c|}
\hline $\begin{array}{l}\text { lodopsin } \stackrel{>-160^{\circ} \mathrm{C}}{\longleftarrow} \\
h v\{\{h v \\
\text { Bathoiodoosin } \\
h v\left\{\begin{array}{l}n v \\
\text { Isoiodopsin }\end{array}\right.\end{array}$ & $\underset{?}{\text { Lumiiodopsin } \rightarrow \rightarrow \longrightarrow} \stackrel{\text { (all-trans)-retinal }}{+}+{ }^{+}$ \\
\hline $\begin{array}{l}\mathrm{N} \text {-lodopsin } \\
h v\}\{h v \\
\mathrm{N} \text {-Bathoiodopsin } \stackrel{.>-160^{\circ} \mathrm{C}}{\longrightarrow} \\
h v\}\{h v \\
\mathrm{N} \text {-isoiodoosin }\end{array}$ & $\mathrm{N}$-Lumiiodopsin $\rightarrow \rightarrow \rightarrow \begin{array}{c}\text { (all-trans)-retinal } \\
+ \\
\text { opsin }\end{array}$ \\
\hline
\end{tabular}

Fig. 5. Photochemical and subsequent thermal reactions of lodopsin and $\mathrm{N}$-iodopsin. Wavy lines: photochemical reactions. Straight lines: thermal reactions observed by the low-temperature spectrophotometry. Dotted lines, reactions which were hardly observed at low temperature.

Recently we have carried out picosecond laser photolysis of iodopsin in order to confirm that bathoiodopsin is a physiological intermediate which is produced at room temperature. We have abserved the formation at room temperature of bathoiodopsin which bleaches to (all-trans)retinal and R-photopsin through several intermediates, Considering the reversion of bathoiodopsin to the original iodopsin observed at low temperature, the conversion of bathoiodopsin into lumiiodopsin would release the chloride ion from the protein. The physiological role of the release of chloride ion must be the subject of future research.

\section{PHOTO-BLEACHING PROCESS OF IODOPSIN}

The bleaching process of iodopsin at room temperature was investigated by means of nanosecond laser photolysis. Like rhodopsin, iodopsin bleached to (all-trans)-retinal and R-photopsin through several thermolabile intermediates. Since we detected four intermediates on the time scale from nanoseconds to milliseconds, we tentatively assigned them as batho, lumi, meta-I and meta-II intermediates of lodopsin. These intermediates formed and decayed faster than the corresponding ones of rhodopsin. In the photo-transduction process in rods, a later intermediate, metarhodopsin 11 , interacts with transducin for transfer of the light signal to the enzymic cascade system. Thus the rate of formation of the meta-ll intermediate is one of the factors determining the rate of generation of the receptor potential. The fact that the putative metaiodopsin II is formed faster than metarhodopsin II may partly explain the difference in latency of the photoresponse between rod and cone.

On the other hand, the photosensitivity of the photoreceptor cell is related to the extent of amplification of the light signal which depends on how many transducins are activated by metarhodopsin II. Thus the lifetime of metarhodopsin II is one of the factors that determine rod photosensitivity. Therefore, our observation that the lifetime of metaiodopsin II is shorter than that of metarhodopsin II would reasonably explain the lower photo-sensitivity of the cone than the rod.

\section{SIGNAL TRANSDUCTION PROCESS IN THE CONE}

We have examined the activation of transducin by irradiation of iodopsin. Since cone transducin has not yet been isolated from retinas, a transducin purified from bovine rod outer segments was used. A purified lodopsin was reconstituted in PC liposomes to which bovine transducin was added. Irradiation of the reconstituted iodopsin remarkably enhanced the binding of GppNHp (a nonhydrolyzable GTP analogue) to transducin. This result indicates that, like rhodopsin, iodopsin exposes a transducin-binding domain only when irradiated (ref. 13). Addition of a small amount of irradiated lodopsin to an excess amount of transducin effectively enhanced the binding. This activation profile was very similar to that produced by irradiation of bovine or chicken rhodopsin, indicating that irradiated iodopsin acts as a catalyst for formation of a GTP-transducin complex (ref. 13). That is, one molecule of a photo-bleaching intermediate of iodopsin can induce the formation of several tens of molecules of the GTP-transducin complex. This result is consistent with our previous observation that CGMP-phosphodiesterase in chicken rod and cone outer segment membranes could be activated by irradiation of iodopsin in the presence of GTP (ref. 14). This step provides the light signal amplification in the cone.

The GTP-transducin complex in turn activates cGMP-phosphodiesterase in rod (the 'on-process' of visual excitation). During the course of successive activation of transducin, catalyzed by metarhodopsin II, serine and threonine residues in the carboxyl-terminal tail of this intermediate are phosphorylated by a specific cyclic-nucleotide-independent kinase, rhodopsin kinase (ref. 15). This phosphorylation shuts off the CGMP-phosphodiesterase activation in rod (the 'off-process' of visual excitation). To examine the possible similarity between the mechanisms in rod and cone, the amino acid sequence of the corresponding region of chicken iodopsin was determined by means of Edman degradation of peptide fragments produced by enzymic proteolysis or CNBr-treatment of the purified iodopsin. The primary structure exhibited prominent homology with that of rhodopsin, having a cluster of serine and threonine residues to be phosphorylated. In fact, a photo-bleaching 
intermediate of iodopsin was phosphorylated by purified bovine rhodopsin kinase; $K_{m}$ and $V_{\text {max }}$ values for the phosphorylation were similar to those for the photobleaching intermediate of rhodopsin (ref. 16). These results suggest that visual pigments in the cone, when they absorb light, would be phosphorylated by the endogenous kinase, and that the phosphorylation might be involved in the shut-off process of the cone transduction.

\section{LOCALIZATION OF CONE PIGMENTS IN THE CHICKEN RETINA}

We have prepared four kinds of monoclonal antibody (mAb) by using purified iodopsin as an antigen (ref. 17). Immunochemical experiments demonstrated that the mAbs were so specific for iodopsin that they recognized neither rhodopsin nor the other three kinds of cone pigments in chicken retina. The specificity of the mAbs was further confirmed by immunohistochemical observation of a whole-mount preparation of chicken retina, in which one rod and five types of cones were present. The cones are morphologically classified into single and double cones. These cones were further identified according to the color of oil droplets; the single cones showed red, yellow, pale-blue, and clear oil droplets, while the double cone had pale-green oil droplets in the principal member and was devoid of oil droplets in the accessory member (ref. 18). These oil droplets contain carotenoids which act as color filters for the light that is absorbed by the cones. 1 mmunohistochemical analysis of the whole-mount preparation of the chicken retina revealed that iodopsin was localized in the outer segments of both the principal and accessory members of the double cone and in the single cone that contained the red oil droplet. Other single cones were not labeled with the mAbs specific for iodopsin (ref. 18),

Interestingly, the mAbs labeled reticular cytoplasmic structures in a position adjacent to the vitreous pole of the paraboloid and to the outer limiting membrane in the cones that bore an immunoreactive outer segment. According to electron microscopic observation, the positive image was localized at the Golgi regions and small vesicular structures (ref. 19). This result indicates a continuous synthesis of iodopsin at the Golgi apparatus.

\section{Acknowledgements}

We would like to express our hearty thanks to a local poulterer, Toripin Co. Ltd., for continuing their kind supply of fresh chicken heads. We also thank Drs. R. S. H. Liu (Univ. of Hawaii), P. A. Hargrave (Univ. of Florida, College of Medicine), T. Oishi (Nara Women's Univ.) and M. Araki (Jichi Medical School) for their fruitful collaborations. This work was supported in part by a Grant-in-Aid for Specially Promoted Research from the Japanese Ministry of Education, Culture and Science, and by a Special Coordination Fund of the Science and Technology Agency of the Japanese Government.

\section{REFERENCES}

1. Y. Shichida, S. Matuoka and T. Yoshizawa, Photobiochem. Photobiophys, 7, 221-228 (1984).

2. G. Wald, P.K. Brown and P.H. Smith, J. Gen, Physiol. 38, $\overline{623-681(1955) .}$

3. A. Knowles, Biochem. Biophys, Res. Commun. 73, 56-62 (1976).

4. L.Y. Fager and R.S. Fager, Exp. Eye Res. 29, 401-408 (1979).

5. H. Matsumoto, F. Tokunaga and T. Yoshizawa, Biochim. Biophys, Acta 404, 300-308 (1975).

6. D.A. Baylor and B. Nunn, Methods in Enzymol. 81, 403-423 (1982),

7. T. Okano, Y. Fukada, I.D. Artamonov and T. Yoshizawa, Biochemistry 28, 8848-8856 (1989).

8. Y. Fukada, T. Okano, Y. Shichida, T. Yoshizawa, A. Trehan, D. Mead, M. Denny, A.E. Asato and R.S.H, Liu, Blochemistry 29, 3133-3140 (1990).

9. H. Matsumoto and T. Yoshizawa, Nature, 258, 523-526 (1975).

10. Y. Shichida, T. Kato, S. Sasayama, Y. Fukada and T. Yoshizawa, Biochemistry 29 5843-5848 (1990).

11. T. Yoshizawa and G. Wald, Nature, 214, 566-571 (1967).

12. Y. Imamoto, H. Kandori, T. Okano, Y. Fukada, Y. Shichida and T. Yoshizawa, Biochemistry 28, $9412-9416$ (1989)

13. Y. Fukada, T. Okano, I.D. Artamonov and T. Yoshizawa, FEBS Lett. 246, 69-72 (1989),

14. Y. Fukada and T. Yoshizawa, FEBS Lett, 149, 117-122 (1982)

15. K. Palczewski, J.H. MCDowell and P.A. Hargrave, Biochemistry 27, 2306-2313 (1989).

16. Y. Fukada, K. Kokame, T. Okano, Y. Shichida, T. Yoshizawa, J.H. McDowell, P.A. Hargrave and K. Palczewski, Biochemistry (in press)

17. Y. Shichida, Y. Taniguchi, O. Kuwata, Y. Fukada, T, Yoshizawa, S. Horiuchi and M. Takeichi, Exp. Eye Res. 48, 281-293 (1989).

18. T. Oishi, A. Kawata, T. Hayashi, Y. Fukada, Y. Snichida and T. Yoshizawa, Cell and Tissue Res. (1990, in press).

19. M. Araki, Y. Fukada, Y. Shichida and T. Yoshizawa, Invest. Ophthalmol. Vis. Sci. (1990, in press). 